\title{
New treatments for metastatic melanoma
}

\author{
Craig Gedye MBChB PhD, David Hogg MD, Marcus Butler MD, Anthony M. Joshua MBBS PhD
}

Competing interests: Craig Gedye has received an honorarium from BristolMyers Squibb. No other competing interests were declared.

This article has been peer reviewed.

Correspondence to: Anthony Joshua, anthony.joshua@uhn.ca

CMAJ 2014. DOI:10.1503 /cmaj.130989
$\mathrm{U}$ ntil 2010, the median survival of patients with metastatic melanoma was about seven months, and only $6 \%$ of patients survived to five years. Chemotherapy with dacarbazine was the standard of care, although it had not been proven to improve patient survival. ${ }^{1}$ Randomized controlled trials have now shown that both kinase inhibitors and immunotherapeutic antibodies can provide dramatic responses and prolong survival compared with chemotherapy (Figure 1A). These new drugs come at the price of novel adverse effects, some of which are life-threatening. In this review, we summarize recent seminal phase III and innovative phase II clinical trials that highlight the opportunities and challenges of these new treatments for metastatic melanoma (Box 1).

\section{How do these new drugs for advanced melanoma work?}

Traditional chemotherapy for melanoma acts by interfering with DNA replication during cell division (Figure 2). Two new classes of drugs possess markedly different mechanisms of action. Kinase inhibitors block inappropriately activated cellsignalling pathways, either at the mutated protein itself (e.g., BRAF) or "downstream" (e.g., a mitogen-activated protein/extracellular signal-regulated kinase kinase $[\mathrm{MEK}]$ inhibitor for an "upstream" NRAS mutation). Immunotherapeutic antibodies enlist the patient's immune system to attack the cancer. By definition, all advanced cancers have escaped the immune system, often by co-opting normal immunoregulatory mechanisms. Immune therapies such as vaccines and inflammatory cytokines have delivered mixed results; ${ }^{2}$ however, spontaneous immune rejection does occur rarely in metastatic melanoma owing to antitumour lymphocytes. ${ }^{3}$ The outcome of the lymphocytetumour cell interaction depends on the balance of immunoregulatory signals, some of which stimulate the lymphocyte to kill the cancer cell, whereas others (e.g., cytotoxic T-lymphocyte-associated protein 4 [CTLA-4] and programmed cell death 1 ligand 1 [PD-L1]) render the lymphocyte inactive. Immunotherapeutic antibodies act by blocking these negative immunoregulatory signals, with the hope that the patient's immune system will recognize and reject the cancer.

The kinase inhibitors vemurafenib, dabrafenib and trametinib, and the immunotherapeutic antibody ipilimumab have approval from Health Canada.

\section{How has the diagnostic approach to metastatic melanoma changed?}

Upfront molecular profiling of each patient's melanoma is now critical for treatment selection, as it identifies mutations in specific genes that are "targetable" for each individual patient. For example if the patient's melanoma is "BRAFmutant" (red line, Figure 1C), a drug targeting mutated BRAF protein or the downstream protein MEK may be useful. Samples of the patient's melanoma must therefore be genotyped in a laboratory certified by the Clinical Laboratory Improvement Amendments.

\section{Why is the MAPK pathway important in melanoma?}

The RAS-RAF-mitogen-activated protein kinase (MAPK) signalling pathway transmits external signals from growth factors and the microenvironment to influence cancer cell growth, differentiation and survival (Figure 2). The $B R A F$ gene encodes the BRAF protein, a critical component of the MAPK pathway. Mutations in $B R A F$ that force "always on" constitutive activity in BRAF are found in about $50 \%$ of melanomas. Whereas 
$20 \%-30 \%$ of $B R A F$ wild-type melanomas are driven by mutations in NRAS, mutations of $M E K$ or KIT are less common. ${ }^{4}$ This cluster of activating mutations in MAPK pathway genes led to the development of drugs to inhibit this pathway.

\section{Are these mutations in melanoma related to clinical features?}

Of melanomas in patients younger than 30 years, $80 \%$ are $B R A F$-mutated, compared with only $20 \%$ in patients older than 70 years. ${ }^{5} B R A F$ mutations are most commonly found in melanomas arising from non-sun-exposed skin, such as the trunk, ${ }^{6}$ whereas $N R A S$-mutated melanomas more often occur in sun-exposed skin, tend to be thicker, have higher growth rates and may have a poorer prognosis. ${ }^{7}$ Amplification or mutation of the KIT gene can drive acral-lentiginous (e.g., soles, palms, nails) and mucosal melanomas. ${ }^{8}$ Some patients with KIT-driven melanoma may respond to kinase inhibitors such as imatinib. ${ }^{9}$ Finally, most patients with uveal (ocular) mel- anoma bear mutations of either GNAQ (47\%) or GNA11 (37\%) genes that also activate the MAPK pathway (Figure 2).

\section{How are kinase inhibitors used to treat BRAF-mutant metastatic melanoma?}

The first specific BRAF inhibitor, vemurafenib, elicited impressive results in patients who had

\section{Box 1: Evidence for this review}

We searched PubMed for clinical trials with the terms "vemurafenib or dabrafenib or trametinib or PLX4032 or GSK2118436 or GSK1120212 or BRAF or MEK or NRAS" and "CTLA-4 or MDX-010 or ipilimumab or tremelimumab or nivolumab or BMS-936558 or BMS-936559 or MK-3475 or PD-1 or PD-L1" and "melanoma." We manually reviewed abstracts from the American Society of Clinical Oncology Annual Meeting. We reviewed 122 articles, excluding articles that lacked mature survival data but including reports on drug toxicity and management.

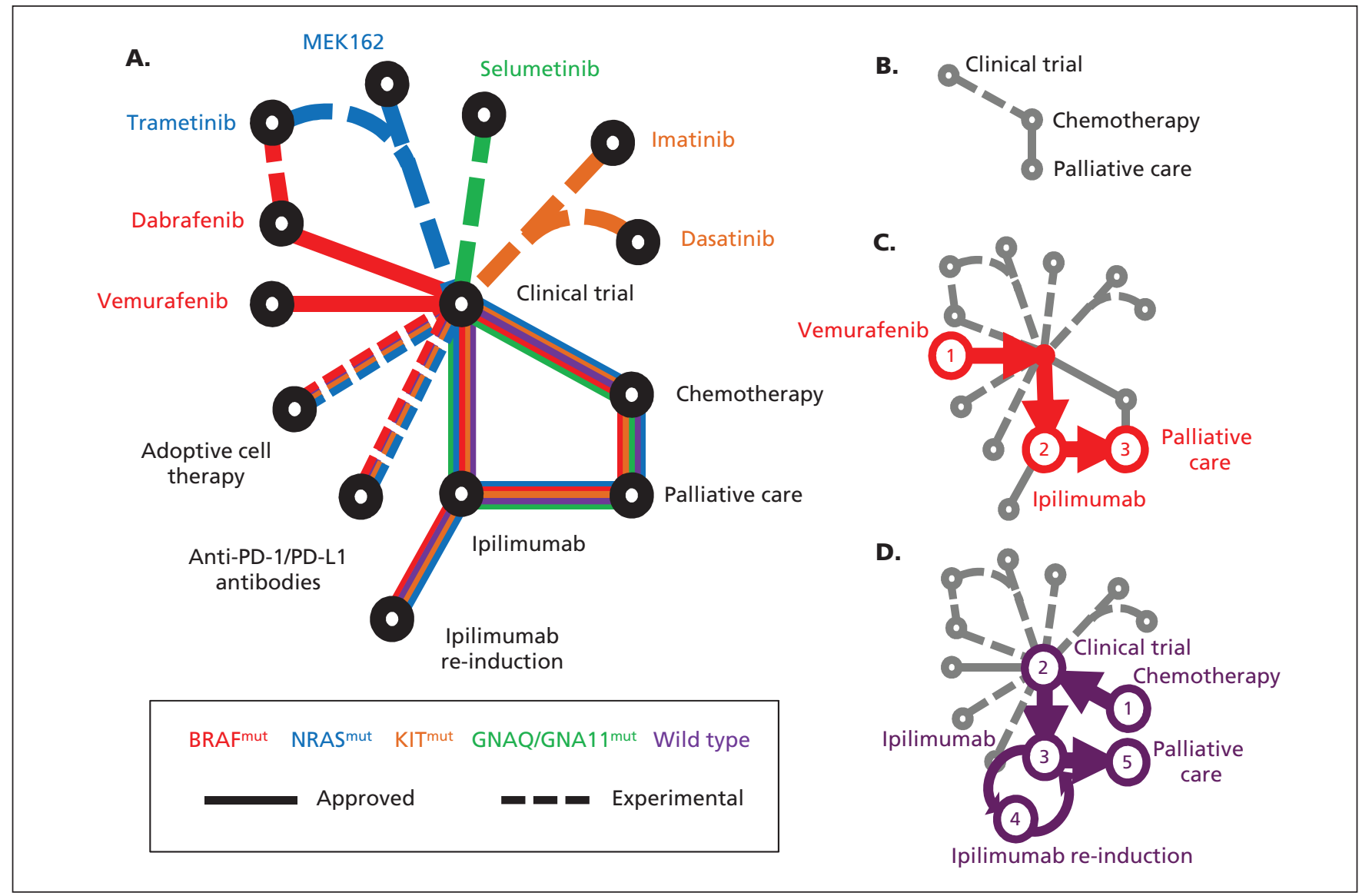

Figure 1: (A) Schematic of newly approved (solid lines) and experimental (dashed lines) treatments for patients with metastatic melanoma. The patient's treatment is personalized according to the underlying driver mutation (e.g., NRAS-mutant in blue). (B) Historically, few treatments were available for melanoma. Examples of treatment progressions are shown for (C) patients with BRAF-mutant melanoma or (D) patients without an identifiable driver mutation (wild type). PD-1 = programmed cell death protein 1, PD-L1 = programmed cell death 1 ligand 1. 
previously received treatment, with about $80 \%$ experiencing tumour shrinkage or stabilization. ${ }^{10}$ In the phase III BRIM-3 trial, 675 patients with treatment-naive $B R A F$-mutant melanoma were randomly assigned to take vemurafenib (960 mg orally, twice daily) or dacarbazine. ${ }^{11}$ Updated follow-up shows a median overall survival of 13.6 months and a one-year survival rate of $56 \%$ for treatment with vemurafenib, versus 9.6 months and 44\%, respectively, for dacarbazine (hazard ratio [HR] $0.62,95 \%$ confidence interval [CI] 0.49-0.77). ${ }^{12}$ In the BREAK-3 trial, a second BRAF inhibitor, dabrafenib (150 mg orally, twice daily) was compared with dacarbazine, with crossover permitted. The progression-free survival was significantly longer with dabrafenib (5.1 v. $2.7 \mathrm{mo}$; HR $0.30,95 \%$ CI $0.18-0.53 ; p<0.0001) .{ }^{13}$

\section{What is the benefit and clinical course of ipilimumab treatment?}

Ipilimumab, an immunotherapeutic antibody, blocks negative immunoregulatory signals from CTLA-4, and was clearly active even in earlyphase trials. ${ }^{14}$ The definitive phase III trial of ipilimumab in metastatic melanoma randomly assigned patients who had previously received treatment to take ipilimumab alone $(3 \mathrm{mg} / \mathrm{kg}$ every three weeks to a maximum of four treatments) against a melanoma vaccine (an appropriate control at the time, now recognized as inactive) or a combination of vaccine and ipilimumab. ${ }^{15}$ Response rates (e.g., tumour shrinkage) were modest (ipilimumab $10.9 \%$, vaccine $1.5 \%$, combination $5.9 \%$ ), but patients receiving ipilimumab alone or in combination had unequivocally improved median overall survival (10.1 and $10.0 \mathrm{mo}$ ), with $22 \%$ and $24 \%$ of patients alive at two years, compared with a median survival of 6.4 months, two-year survival of $14 \%$ in patients who received vaccine alone (HR for overall survival for ipilimumab alone v. vaccine $0.66,95 \%$ CI $0.51-0.87 ; p=0.003$ ). Ipilimumab was the first therapy to show a statistically significant and clinically meaningful survival benefit in metastatic melanoma.

In practice, four infusions of ipilimumab ( $3 \mathrm{mg} / \mathrm{kg}$ ) are given every three weeks. About $10 \%-15 \%$ of patients experience tumour shrinkage; another $20 \%-30 \%$ benefit with stable disease. ${ }^{15}$ Responses are slow, often weeks or months after treatment, and a minority of patients may have apparent early progression but experience a delayed response. These slow but meaningful benefits have shifted our traditional treatment goal from tumour shrinkage to

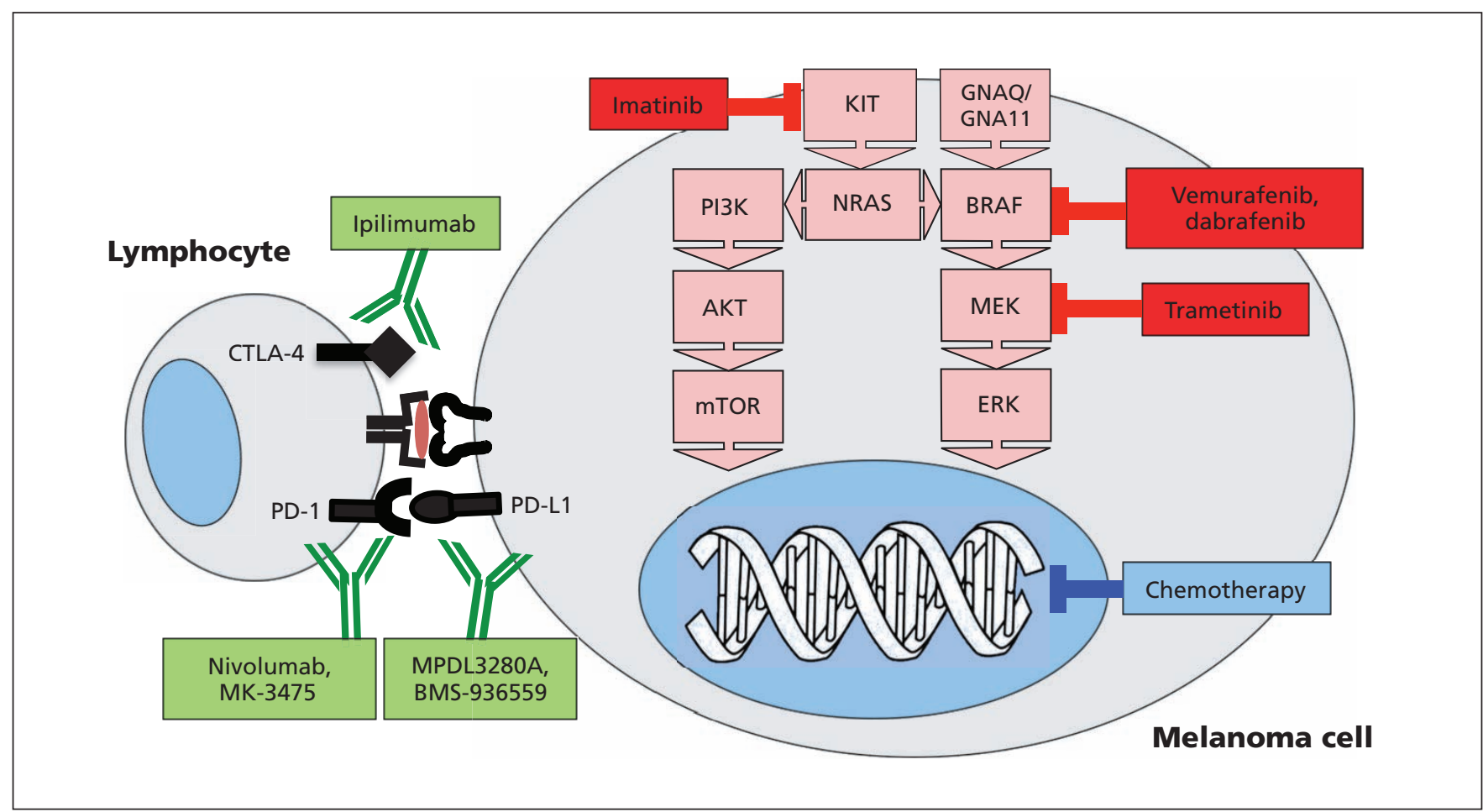

Figure 2: Immunotherapeutic antibodies target immune regulatory proteins on the surfaces of melanoma and lymphocyte cells (green), while kinase inhibitors (red) block mutated mitogen-activated protein kinase (MAPK) pathways. Traditional cytotoxic chemotherapy targets DNA replication (blue). CTLA-4 = cytotoxic T-lymphocyte-associated protein 4, ERK = extracellular signal-regulated kinase, MEK = mitogen-activated protein/ERK kinase, $\mathrm{mTOR}=$ mammalian target of rapamycin, PD-1 = programmed cell death protein 1, PD-L1 = programmed cell death 1 ligand 1. 
control. ${ }^{16}$ A substantial minority (about 25\%) of patients from early clinical trials are still alive after five years, which emphasizes the longterm benefits of ipilimumab. ${ }^{17}$ Ongoing trials are investigating the optimal dose, combination and sequencing of ipilimumab.

\section{How are the adverse effects of BRAF inhibitors and ipilimumab managed?}

Common adverse effects of BRAF inhibitors include dry skin, rash, fatigue, arthralgia, myalgia, elevations of liver enzyme levels, nausea, alopecia, hand-foot syndrome, photosensitivity (vemurafenib; ${ }^{11} 30+$ ultraviolet A sunscreen required) and fever (dabrafenib $\left.{ }^{13}\right)$. Most toxicity is manageable, although dose interruptions and reductions are required in $40 \%-50 \%$ of patients. ${ }^{11}$ The development of cutaneous squamous cell carcinoma, often keratoacanthoma type, in up to $25 \%$ of patients is a unique toxicity of BRAF inhibitors. Dermatologic surveillance with excision or cryotherapy if necessary effectively manages this novel adverse effect.

Immediate toxicity with ipilimumab is minimal; the common toxicities are delayed immunerelated adverse events. In contrast to adverse events from chemotherapy, immune-related adverse events occur weeks or months after the first dose of ipilimumab, even after completion of therapy. Immune-related adverse events have a prolonged duration and a propensity to recur or worsen despite initial relief from immunosuppressive treatment. At least one immunerelated adverse event will occur in $70 \%$ of patients, but they are severe (grade 3 or 4 ) in about $15 \%-25 \%$ of patients. $^{15}$

The most dangerous immune-related adverse event associated with ipilimumab is colitis, ${ }^{18}$ which may present initially with mild diarrhea, bloating and abdominal discomfort. If left unrecognized and untreated, this adverse event can progress to bowel perforation, peritonitis and death. Unless diarrhea is easily controlled with loperamide, the treating physician should rapidly escalate to high-dose oral or intravenous corticosteroids. Inpatient management may be required, and rare patients may require the antitumour necrosis factor- $\alpha$ antibody infliximab. ${ }^{19}$ Rash is relatively common but usually responds to topical treatments. Hepatic toxicity is uncommon and usually asymptomatic, but if severe usually responds to steroids. Endocrinopathies (e.g., hypophysitis) are less common, often delayed and may require long-term hormone replacement therapy. ${ }^{20}$

\section{What options are available when treatment fails or disease relapses?}

Unfortunately, metastatic melanoma almost always progresses during treatment with a BRAF inhibitor, with regrowth of existing lesions and development of new metastases (Figure 3). Median time to progression is about six months. A broad variety of mechanisms have been discovered to cause failure of BRAF inhibitors, including activation of alternate signalling pathways, ${ }^{21}$ activation of other RAS or RAF proteins, ${ }^{22}$ or downstream mutations in the $M E K$ gene..$^{23}$

If a patient benefited from ipilimumab (shrinkage or stable disease) but the disease subsequently progresses, then treatment with a further four doses of ipilimumab can regain control of disease in $13 \%-37 \%$ of patients. ${ }^{24}$ Prior severe immune-related adverse events preclude retreatment with ipilimumab. If the patient's disease

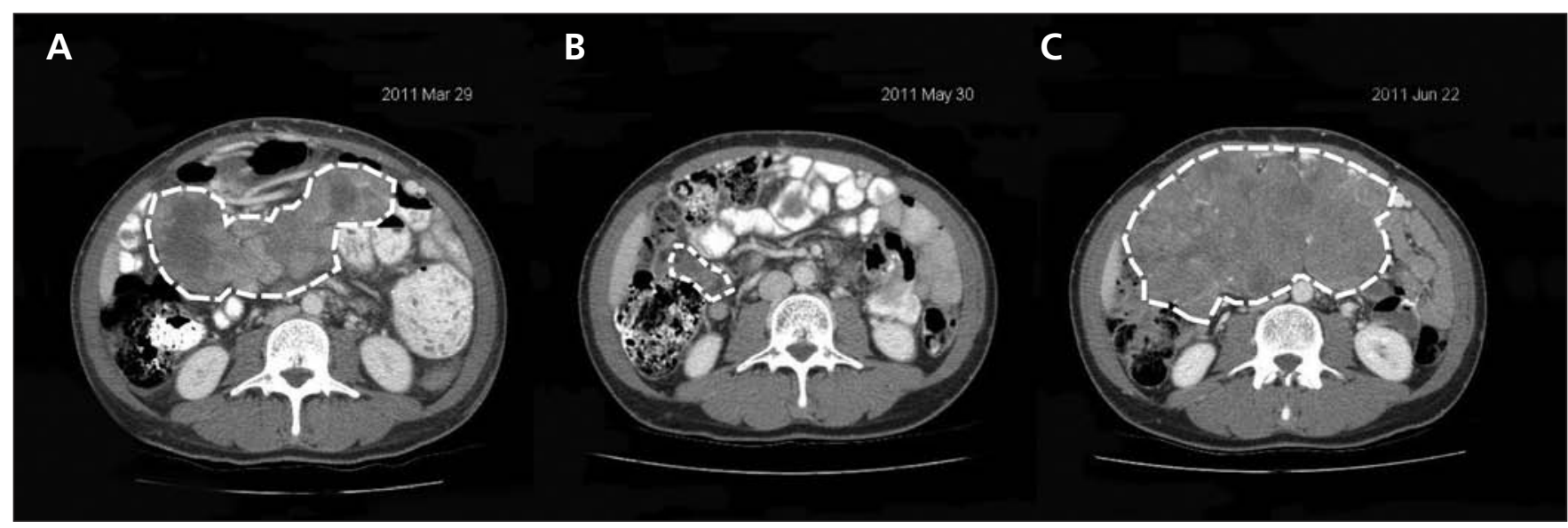

Figure 3: Responses to BRAF inhibitors can be dramatic, but relapses are almost invariable and can be equally rapid. Abdominal computed tomographic scans showing peritoneal metastases (dashed white lines) before treatment (A), and after two months (B) and three months (C) of treatment with vemurafenib. 
progresses through treatment with ipilimumab, then kinase inhibitors, chemotherapy or a clinical trial are appropriate options (Figure 1).

\section{Which patients should receive these therapies and in what order?}

Ipilimumab (second-line treatment only) and vemurafenib are reimbursed in most provinces (excluding the Atlantic provinces and the territories).

Patients with rapidly progressive, symptomatic disease might best start with an appropriate kinase inhibitor, then move on to immunotherapy (Figure 1C), whereas patients with more indolent disease would likely benefit from an immunotherapeutic antibody earlier (Figure 1D). Responses to ipilimumab take up to six months, so patients with rapidly progressive disease may not survive to benefit from ipilimumab. Kinase inhibitors must be personalized; if a mutation is absent, the drug will provide no benefit and may even be harmful. ${ }^{10}$

\section{What other novel inhibitors and immunotherapy treatments are being developed?}

Trametinib is an inhibitor of MEK, downstream of BRAF and NRAS in the MAPK pathway (Figure 2). In a randomized phase I/II trial,

\section{Box 2: Unanswered questions}

- Determining the correct treatment sequence or combination of these new drugs is under intense investigation, but novel combinations must be investigated only within well-conducted clinical trials (e.g., the combination of vemurafenib and ipilimumab has caused unexpectedly and unacceptably severe hepatotoxicity ${ }^{40}$ ). Sequencing choices are limited in practice because ipilimumab is reimbursed only for second-line treatment in Canada.

- There are no pretreatment biomarkers to predict response to immunotherapeutic antibodies. If unresponsive patients could be identified a priori, it would avoid the cost and toxicity of these drugs in these patients.

- Thin $(<2 \mathrm{~mm})$ primary melanomas are most often cured by surgery alone. Thick primary melanoma (stage II) can unfortunately recur in $20 \%-50 \%$ of patients. Both ipilimumab and BRAF inhibitors are being investigated in adjuvant clinical trials with the hope of increasing the cure rates of high-risk primary melanomas. patients with $B R A F$-mutant melanomas were randomly assigned to receive dabrafenib alone or in combination with trametinib. ${ }^{25}$ Responses occurred among $76 \%$ of patients who received the combination, versus $54 \%$ who received dabrafenib alone. The median progression-free survival was 9.4 months versus 5.8 months, respectively (HR 0.39 , 95\% CI $0.25-0.62 ; p<$ 0.001). Although fever was more frequent, other adverse effects seemed attenuated, with severe ( $\geq$ grade 3 ) adverse effects in only $5 \%$ of patients and a reduced frequency of squamous carcinomas and rash among patients taking the combination.

Activating mutations in NRAS are being targeted in clinical trials by downstream inhibition of MEK. ${ }^{26}$ Among 13 patients with NRAS-mutant melanomas who received the MEK inhibitor MEK162, three had a partial response and four had stable disease. ${ }^{27}$ Finally, the MEK inhibitor selumetinib improved progression-free survival compared with chemotherapy among patients with metastatic uveal melanoma. ${ }^{28}$

Adoptive cell immunotherapy involves ex vivo expansion of antitumour T lymphocytes, which are later infused back into patients. The advantage of this approach is that antitumour T lymphocytes can be expanded to large numbers in a laboratory environment without the negative immunoregulatory environment that exists in vivo. Approaches include the generation of tumour antigen-specific $\mathrm{T}$ cell lines and clones $^{29}$ or gene modification of lymphocytes to make them more specific for cancer cells. ${ }^{30}$ Tumour-infiltrating lymphocytes can be isolated and expanded from patients' tumours and reinfused in combination with high-dose chemotherapy and interleukin-2, resulting in high response rates in selected patients. ${ }^{31}$ Efforts to extend these treatments to centres around the world are ongoing. ${ }^{32}$

Programmed cell death protein 1 (PD-1) and programmed cell death 1 ligand 1 (PD-L1) are another pair of immunoregulatory molecules (Figure 2). ${ }^{33}$ In phase I trials, both fully human antiPD-1 antibodies (nivolumab, BMS-936558; and MK-3475) and an anti-PD-L1 blocking antibody (BMS-936559) were found to be safe and remarkably effective. ${ }^{34,35}$ Among patients with melanoma given nivolumab, $28 \%$ experienced a partial response, with stable disease seen in a further $6 \%$, and many responses were prolonged and ongoing, even among patients with visceral and bony metastases. ${ }^{36,37}$ Severe immune-related adverse events developed among $13 \%$ of patients, including hypophysitis, hepatitis, colitis, pneumonitis and thyroiditis.

A second PD-1 antibody, MK-3475, has re- 
cently shown rapid and prolonged responses among up to $52 \%$ of patients with melanoma, both who had received prior treatment with ipilimumab and those who had not. ${ }^{38}$ Of responding patients, $81 \%$ were still receiving treatment at a median of 11 months ${ }^{38}$ Finally, the combination of nivolumab and ipilimumab has shown benefit in two-thirds of patients, with substantial (> 80\%) tumour shrinkage seen in $53 \%{ }^{39}$ Severe immune-related adverse events were seen among half of patients, but were not more frequent or severe than those experienced by patients receiving ipilimumab monotherapy in this early-phase clinical trial. ${ }^{39}$

Unanswered research questions are addressed in Box 2.

\section{Conclusion}

Novel treatments for metastatic melanoma are providing a vast improvement over traditional chemotherapy, albeit with novel and potentially serious adverse effects. Further agents and combinations are being studied in clinical trials and will likely further improve the lives of patients with metastatic melanoma.

\section{References}

1. Barth A, Wanek LA, Morton DL. Prognostic factors in 1,521 melanoma patients with distant metastases. J Am Coll Surg 1995;181:193-201.

2. Cebon J, Gedye C, John T, et al. Immunotherapy of advanced or metastatic melanoma. Clin Adv Hematol Oncol 2007;5:994-1006.

3. Halliday GM, Patel A, Hunt MJ, et al. Spontaneous regression of human melanoma/nonmelanoma skin cancer: association with infiltrating CD4+ T cells. World J Surg 1995;19:352-8.

4. Curtin JA, Fridlyand J, Kageshita T, et al. Distinct sets of genetic alterations in melanoma. $N$ Engl J Med 2005;353:2135-47.

5. Bauer J, Büttner P, Murali R, et al. BRAF mutations in cutaneous melanoma are independently associated with age, anatomic site of the primary tumor, and the degree of solar elastosis at the primary tumor site. Pigment Cell Melanoma Res 2011; 24:345-51.

6. Long GV, Menzies AM, Nagrial AM, et al. Prognostic and clinicopathologic associations of oncogenic BRAF in metastatic melanoma. J Clin Oncol 2011;29:1239-46.

7. Jakob JA, Bassett RL Jr, Ng CS, et al. NRAS mutation status is an independent prognostic factor in metastatic melanoma. Cancer 2012;118:4014-23.

8. Omholt K, Grafström E, Kanter-Lewensohn L, et al. KIT pathway alterations in mucosal melanomas of the vulva and other sites. Clin Cancer Res 2011;17:3933-42.

9. Guo J, Si L, Kong Y, et al. Phase II, open-label, single-arm trial of imatinib mesylate in patients with metastatic melanoma harboring c-Kit mutation or amplification. J Clin Oncol 2011;29:2904-9.

10. Flaherty KT, Puzanov I, Kim KB, et al. Inhibition of mutated, activated BRAF in metastatic melanoma. N Engl J Med 2010; 363:809-19.

11. Chapman PB, Hauschild A, Robert C, et al. Improved survival with vemurafenib in melanoma with BRAF V600E mutation. N Engl J Med 2011;364:2507-16.

12. Chapman PB, Hauschild A, Robert C, et al. Updated overall survival (OS) results for BRIM-3, a phase III randomized, open-label, multicenter trial comparing BRAF inhibitor vemurafenib (VEM) with dacarbazine (DTIC) in previously untreated patients with BRAFV600E-mutated melanoma. 2012 ASCO Annual Meeting Proceedings. J Clin Oncol 2012;30(15 Suppl):8502.

13. Hauschild A, Grob JJ, Demidov LV, et al. Phase III, randomized, open-label, multicenter trial (BREAK-3) comparing the BRAF kinase inhibitor dabrafenib (GSK2118436) with dacarbazine (DTIC) in patients with BRAFV600E-mutated melanoma. 2012
ASCO Annual Meeting Proceedings. J Clin Oncol 2012;30(18 Suppl):LBA8500.

14. Phan GQ, Yang JC, Sherry RM, et al. Cancer regression and autoimmunity induced by cytotoxic $\mathrm{T}$ lymphocyte-associated antigen 4 blockade in patients with metastatic melanoma. Proc Natl Acad Sci U S A 2003;100:8372-7.

15. Hodi FS, O'Day SJ, McDermott DF, et al. Improved survival with ipilimumab in patients with metastatic melanoma. $N$ Engl $J$ Med 2010;363:711-23.

16. Wolchok JD, Hoos A, O'Day S, et al. Guidelines for the evaluation of immune therapy activity in solid tumors: immune-related response criteria. Clin Cancer Res 2009;15:7412-20.

17. Lebbe C, Weber JS, Maio M, et al. Five-year survival rates for patients (PTS) with metastatic melanoma (MM) treated with ipilimumab (IPI) in phase II trials [abstract]. Proceedings of the 2012 European Oncology Congress; 2012 Sept. 28 to Oct. 2; Vienna, Austria. Ann Oncol 2012;23(Suppl 9):9053.

18. Berman D, Parker SM, Siegel J, et al. Blockade of cytotoxic Tlymphocyte antigen-4 by ipilimumab results in dysregulation of gastrointestinal immunity in patients with advanced melanoma. Cancer Immun 2010;10:11.

19. Minor DR, Chin K, Kashani-Sabet M. Infliximab in the treatment of anti-CTLA4 antibody (ipilimumab) induced immunerelated colitis. Cancer Biother Radiopharm 2009;24:321-5.

20. Chin K, Ibrahim R, Berman D, et al. Treatment guidelines for the management of immune-related adverse events in patients treated with ipilimumab, an anti-CTLA4 therapy [abstract]. Ann Oncol 2008;19(Suppl 8):787.

21. Wilson TR, Fridlyand J, Yan Y, et al. Widespread potential for growth-factor-driven resistance to anticancer kinase inhibitors. Nature 2012;487:505-9.

22. Villanueva J, Vultur A, Lee JT, et al. Acquired resistance to BRAF inhibitors mediated by a RAF kinase switch in melanoma can be overcome by cotargeting MEK and IGF-1R/PI3K. Cancer Cell 2010;18:683-95.

23. Wagle N, Emery C, Berger MF, et al. Dissecting therapeutic resistance to RAF inhibition in melanoma by tumor genomic profiling. J Clin Oncol 2011;29:3085-96.

24. Robert C, Schadendorf D, Messina M, et al.; MDX010-20 investigators. Efficacy and safety of retreatment with ipilimumab in patients with pretreated advanced melanoma who progressed after initially achieving disease control. Clin Cancer Res 2013; 19:2232-9.

25. Flaherty KT, Infante JR, Daud A, et al. Combined BRAF and MEK inhibition in melanoma with BRAF V600 mutations. N Engl J Med 2012;367:1694-703.

26. Jaiswal BS, Janakiraman V, Kljavin NM, et al. Combined targeting of BRAF and CRAF or BRAF and PI3K effector pathways is required for efficacy in NRAS mutant tumors. PLOS ONE 2009;4:e5717.

27. Ascierto PA, Berking C, Agarwala SS, et al. Efficacy and safety of oral MEK162 in patients with locally advanced and unresectable or metastatic cutaneous melanoma harboring BRAFV600 or NRAS mutations. 2012 ASCO Annual Meeting Proceedings. J Clin Oncol 2012;30(15 Suppl):8511.

28. Richard D, Carvajal JAS, Fernando Quevedo, et al. Phase II study of selumetinib (sel) versus temozolomide (TMZ) in gnaq/Gna11 (Gq/11) mutant (mut) uveal melanoma (UM). 2013 ASCO Annual Meeting Proceedings. J Clin Oncol 2013;31(18 Suppl):CRA9003.

29. Butler MO, Friedlander P, Milstein MI, et al. Establishment of antitumor memory in humans using in vitro-educated CD8+ T cells. Sci Transl Med 2011;3:80ra34.

30. Grupp SA, Kalos M, Barrett D, et al. Chimeric antigen receptormodified T cells for acute lymphoid leukemia. $N$ Engl J Med 2013;368:1509-18.

31. Dudley ME, Yang JC, Sherry R, et al. Adoptive cell therapy for patients with metastatic melanoma: evaluation of intensive myeloablative chemoradiation preparative regimens. J Clin Oncol 2008;26:5233-9.

32. Besser MJ, Shapira-Frommer R, Itzhaki O, et al. Adoptive transfer of tumor-infiltrating lymphocytes in patients with metastatic melanoma: intent-to-treat analysis and efficacy after failure to prior immunotherapies. Clin Cancer Res 2013;19:4792-800.

33. Dong H, Strome SE, Salomao DR, et al. Tumor-associated B7$\mathrm{H} 1$ promotes T-cell apoptosis: a potential mechanism of immune evasion. Nat Med 2002;8:793-800.

34. Brahmer JR, Drake CG, Wollner I, et al. Phase I study of singleagent anti-programmed death-1 (MDX-1106) in refractory solid tumors: safety, clinical activity, pharmacodynamics, and immunologic correlates. J Clin Oncol 2010;28:3167-75.

35. Brahmer JR, Tykodi SS, Chow LQM, et al. Safety and activity of Anti-PD-L1 antibody in patients with advanced cancer. N Engl J Med 2012;366:2455-65. 
36. Hodi FS, Sznol M, McDermott DF, et al. Clinical activity and safety of anti-PD-1 (BMS-936558, MDX-1106) in patients with advanced melanoma (MEL). 2012 ASCO Annual Meeting Proceedings. J Clin Oncol 2012;30(15 Suppl):8507.

37. Topalian SL, Hodi FS, Brahmer JR, et al. Safety, activity, and immune correlates of anti-PD-1 antibody in cancer. $N$ Engl J Med 2012;366:2443-54

38. Hamid O, Robert C, Daud A, et al. Safety and tumor responses with lambrolizumab (anti-PD-1) in melanoma. $N$ Engl J Med 2013;369:134-44

39. Wolchok JD, Kluger H, Callahan MK, et al. Nivolumab plus ipilimumab in advanced melanoma. N Engl J Med 2013;369: 122-33.
40. Ribas A, Hodi FS, Callahan M, et al. Hepatotoxicity with combination of vemurafenib and ipilimumab. N Engl J Med 2013; $368: 1365-6$

Affiliation: The Department of Medical Oncology and Hematology, Princess Margaret Cancer Centre, Toronto, Ont.

Contributors: Craig Gedye drafted the article and contributed substantially to the conception and design. David Hogg, Anthony Joshua and Marcus Butler revised the manuscript critically for important intellectual content. All of the authors approved the version submitted for publication.

\section{Resources}

Patient resources

- Melanoma Network of Canada; www.melanomanetwork.ca

- Save your Skin Foundation; www.saveyourskin.ca

- David Cornfield Melanoma Fund; http://dcmf.ca

- National Comprehensive Cancer Network. NCCN Guidelines for Patients; www.nccn.org/patients/guidelines/melanoma/index.html

- My Cancer Genome; www.mycancergenome.org/content/disease/melanoma

Physician resources

- National Comprehensive Cancer Network. NCCN Guidelines for Physicians - Melanoma (free subscription required); www.nccn.org/professionals/physician_gls/pdf/melanoma.pdf

- Cancer Care Ontario. Melanoma Evidence-based Series and Practice Guidelines; www.cancercare.on.ca/toolbox/qualityguidelines/diseasesite/melanoma-ebs/

- Clinical Practice Guidelines for the Management of Melanoma in Australia and New Zealand; www.nhmrc.gov.au/_files_nhmrc/publications/attachments/cp111.pdf 